\title{
Evidence, Temperature, and the Laws of Thermodynamics
}

\author{
Veronica J. Vieland \\ The Research Institute at Nationwide Children's Hospital and The Ohio State University, Columbus, Ohio, USA
}

\section{Key Words}

Likelihood principle $\cdot$ Statistical evidence $\cdot$ Temperature .

Thermodynamics

\begin{abstract}
A primary purpose of statistical analysis in genetics is the measurement of the strength of evidence for or against hypotheses. As with any type of measurement, a properly calibrated measurement scale is necessary if we want to be able to meaningfully compare degrees of evidence across genetic data sets, across different types of genetic studies and/or across distinct experimental modalities. In previous papers in this journal and elsewhere, my colleagues and I have argued that geneticists ought to care about the scale on which statistical evidence is measured, and we have proposed the Kelvin temperature scale as a template for a context-independent measurement scale for statistical evidence. Moreover, we have claimed that, mathematically speaking, evidence and temperature may be one and the same thing. On first blush, this might seem absurd. Temperature is a property of systems following certain laws of nature (in particular, the 1st and 2nd Law of Thermodynamics) involving very physical quantities (e.g., energy) and processes (e.g., mechanical work). But what do the laws of thermodynamics have to do with statistical systems? Here I address that question.

(c) 2014 S. Karger AG, Basel
\end{abstract}

\section{Introduction}

This is a paper about the 1st and 2nd Law of Thermodynamics. So the question 'what is it doing in a genetics journal?' presents itself naturally. Previously in this journal [1-4] and elsewhere [5, 6], my colleagues and I have advocated the need for an absolute, context-independent scale for measurement of evidence in statistical genetics and other fields. We have illustrated situations in which the lack of such a measure leads to erroneous inference, e.g., the conclusion that an original linkage or association report was a false positive when in fact subsequent data support the original finding. And we have begun to develop a theoretical foundation for a properly calibrated scale using the mathematics of thermodynamics as a template. Here I fill in a lacuna in the framework as developed thus far, namely, the lack of any obvious connection between the laws of thermodynamics and the behavior of statistical systems per se.

It is well-known that important connections exist between statistical mechanics and information theory. For instance, it has been widely recognized for some time that the concepts of entropy developed by Gibbs and others have, at least under some well-circumscribed conditions, the same form as Shannon's measure of average information or Shannon entropy [7]. The information-entropy connection has been further developed in connection

\section{KARGER}

E-Mail karger@karger.com

www.karger.com/hhe (c) 2014 S. Karger AG, Basel

0001-5652/14/0784-0153\$39.50/0
Veronica Vieland

Columbus Children's Research Institute

700 Children's Drive, WA-4021

Columbus, OH 43205 (USA)

E-Mail veronica.vieland@ nationwidechildrens.org 
with Gibbs' paradox [8] and Maxwell's demon [9] and elsewhere. To a physicist, the link between physics and information theory can be made by treating information in its physical embodiment, i.e., by recognizing that the extraction of information requires physical action, and then describing that action in familiar physical terms [10]. This is one way to reconcile the observation of identical bits of mathematics governing two topics - physics and information theory - that seem to be very distinct metaphysically. This tack has been taken recently by physicists Duncan and Semura [11, 12], who have proposed a wholly information-based version of the 2nd Law of Thermodynamics, but again stressing the physical embodiment of the type of information covered by this law. (See also Jaynes [8] and Caticha [13, 14] for other purely probabilistic, or information-based, accounts of the 2 nd Law of Thermodynamics and its relationship to statistical inference.) Note that all of these papers are concerned with the 2nd Law of Thermodynamics, not the 1st. But thermodynamics as a whole requires both laws.

Here I show how both the 1st and 2nd Law of Thermodynamics can be interpreted without reference to the physical manifestation of information or to energy in its physical form. This clears the path to reinterpreting temperature itself as a purely mathematical entity, one which, as I shall illustrate, has a central role to play in statistical inference.

The remainder of this paper is organized as follows: I begin with a brief review of some key characteristics of evidence, and then I discuss the 1st Law of Thermodynamics, deriving a formal (purely mathematical) connection with the familiar likelihood principle; the 2nd Law of Thermodynamics, relating it to a principle concerning information loss inherent in the use of likelihood; and the link between these two principles in a purely statistical, non-physical, system. If we assume that this link has the same form in this new information-dynamic system as it does in thermodynamics itself, then we arrive at the very surprising conclusion that thermodynamic ' $T$ ' has an important, purely information-based interpretation in addition to its interpretation as temperature, i.e., as the strength of statistical evidence, in a sense to be made clear.

\section{Evidence}

Statistical evidence is often characterized as a relationship between data and hypotheses in the context of a known probability model, and I will adhere to this high- level description. (Of course when the model is not known, we also have to deal with issues associated with approximating models; I do not consider this complication here.) However, rather than adopting a particular formal definition of evidence from the outset, the objective here is to derive a measure of evidence that has certain desirable properties. I begin by briefly reviewing some properties of statistical evidence, in a simple setting in which our intuitions are clear. Insofar as these properties are intrinsic to what we mean when we talk about evidence, they are properties that any formal treatment of evidence would need to recapitulate.

Consider a series of $n$ independent coin tosses of which $x$ land heads $(\mathrm{H})$. Let the probability that the coin lands $\mathrm{H}$ be $\theta$. And consider the two hypotheses 'coin is biased towards tails $(\bar{H})$ ' $(\theta<1 / 2)$ versus 'coin is fair' (i.e., the coin lands $\bar{H}$ and $H$ with equal probabilities, or $\theta=1 / 2$; I use the notation $\overline{\mathrm{H}}$ to designate tails, since the more obvious choice ' $\mathrm{T}$ ' is already being used for absolute temperature). Note that this set-up corresponds to linkage analysis based on fully informative gametes with $\theta$ representing the recombination fraction, and in statistical terms, it represents a comparison between a simple and a composite hypothesis. I shall assume this form of comparison throughout, but comment on it in 'Conclusions'. What follows are some thought experiments appealing to the reader's statistical intuition.

Suppose that on repeated tosses the coin consistently lands $\mathrm{H}$ (approximately) 5\% of the time. Clearly as the number of tosses $n$ increases, if $x / n$ remains around 5\%, the evidence that the coin is biased increases. Now consider a fixed number of tosses $n$, but allow $x / n$ to increase from $5 \%$ upwards. Here the evidence in favor of bias clearly decreases as the proportion $x / n$ increases in the direction of $1 \frac{1}{2}$. (If you are not sure about this, note that at some point, as $x / n$ approaches $1 / 2$ the evidence will switch to favoring 'no bias;' therefore the evidence for bias must be diminishing as $x / n$ increases up to that point.) These two features together entail a third, somewhat more abstract property. Suppose now that we hold the evidence constant (without defining it or saying how to calculate it). If $x / n$ starts at 0.05 , what must happen, as $n$ increases, in order to prevent the evidence itself from increasing? If the previous two properties hold, then it follows that in this scenario, $x / n$ must increase; otherwise the evidence would increase as $n$ increases rather than remaining constant. Thus the three quantities $n, x$ and evidence $e$ enter into an equation of state, in which holding any one of the three constant, while allowing a second to change, necessitates a compensatory change in the third. Here $e$ itself is 
simply defined as the third fundamental entity in the set. Note that we are not doing any actual calculations here, but only considering patterns of evidence as a function of changes in data.

I note two additional and very important properties of $e$ up front. First, for a given $n$, as $x / n$ increases from some very small value towards $1 / 2$, the evidence for 'bias' at first decreases, but the evidence remains in favor of bias up to some particular value of $x / n$. Beyond that value, however, as $x / n$ continues to approach $1 / 2$ from the left, the evidence switches to favoring 'no bias'. I refer to the value of $x / n$ at which this switch occurs as the transition point $(\operatorname{TrP})$. To the right of the $\operatorname{TrP}$, as $x / n$ continues to increase beyond this point up to $x=n / 2$, the evidence for 'no bias' increases. Figure 1 illustrates this pattern. Note that the existence of the $\mathrm{TrP}$ in and of itself does not change the focus on evidence; in particular, here I am not interested in testing whether $\theta$ is to the left or the right. (Statistical intuition also suggests that $\operatorname{TrP}$ itself ought to move towards $x=n / 2$ as $n$ increases, so that even a small deviation from $x=n / 2$ would correspond to evidence against $\theta=1 / 2$ for very large $n$. A mirror-image set of properties would also occur as $x / n$ increases to the right of $\theta=1 / 2$, but by restricting attention to the one-sided hypothesis contrast, we forego the need to consider $\theta>1 / 2$.)

Finally, note that the same quantity of new data $(n, x)$ seems to have a smaller impact on our intuitive assessment of the evidence, the larger the starting value of $n$, or equivalently, the stronger evidence $e$ before consideration of the new data. E.g., suppose we have already tossed the coin 1,000 times and observed $x=0$. We will all agree that this is overwhelming evidence of a biased coin. If we toss the coin an additional 5 times and observe $0 \mathrm{H}$, the new data $(5,0)$ change the strength of the evidence hardly at all. However, had we started with just 2 tosses with $0 \mathrm{H}$, then added the same new data $(5,0)$, the evidence in favor of bias would have changed quite a bit. The initial set (2, 0 ) gives only very slight evidence for bias, but by the time we have observed $(7,0)$, we would be far more suspicious that the coin is not fair. Despite the purely qualitative nature of this example, it illustrates the key point that evidence is not equivalent to or even inherent in data per se: the evidence conveyed by new data depends on the context in which the new data are observed.

Table 1 summarizes these key properties of evidence. They play no particular role in the next two sections, but they become critically important in The Missing Link. In the mean time, they serve to motivate the use of the qualifier 'evidential' in some of the following text. One last prefatory note: up to this point and throughout the fol-

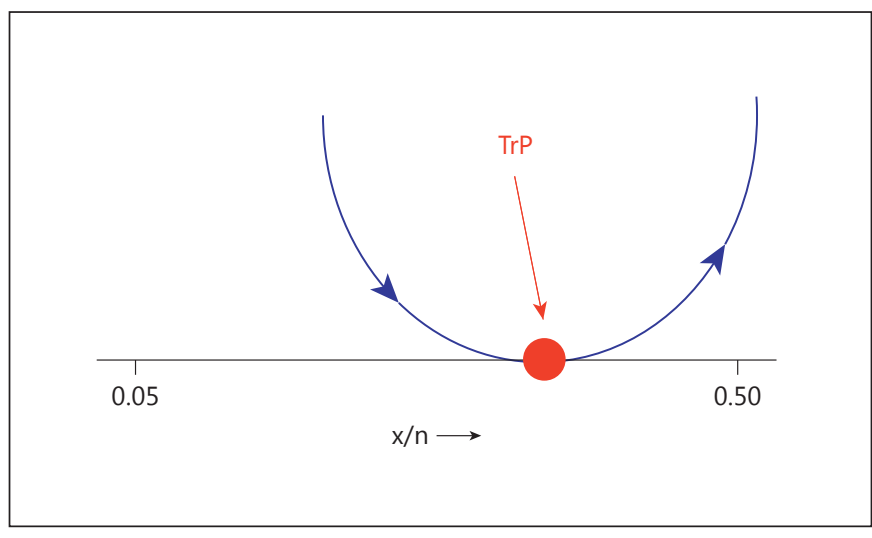

Fig. 1. Schematic illustration of the behavior of evidence $e$ (blue curve) around the TrP, as a function of increasing $x / n$ for fixed $n$.

Table 1. Four defining properties of evidence $e$

\begin{tabular}{|c|c|c|c|c|c|c|}
\hline \multirow[t]{2}{*}{ Property } & \multicolumn{3}{|c|}{$\begin{array}{l}\text { To the left of the } \operatorname{TrP} \\
\text { (evidence for bias) }\end{array}$} & \multicolumn{3}{|c|}{$\begin{array}{l}\text { To the right of the } \operatorname{TrP} \\
\text { (evidence against bias) }\end{array}$} \\
\hline & $n$ & $x / n$ & $e$ & $n$ & $x / n$ & $e$ \\
\hline P1 & $\uparrow$ & $=$ & $\nearrow$ & $\uparrow$ & $=$ & $\nearrow$ \\
\hline $\mathrm{P} 2$ & $=$ & $\uparrow$ & $\searrow$ & $=$ & $\uparrow$ & $\nearrow$ \\
\hline P3 & $\uparrow$ & $\nearrow$ & $=$ & $\uparrow$ & $\searrow$ & $=$ \\
\hline $\mathrm{P} 4$ & \multicolumn{6}{|c|}{$\Delta e$ with new data depends on previous data } \\
\hline
\end{tabular}

Patterns are shown corresponding to the thought experiments described in the text, in which two of the three quantities $(n, x / n$, e) follow a stipulated pattern and our intuition regarding the behavior of the third quantity is recorded. For $\mathrm{P} 1-\mathrm{P} 3$, $\uparrow$ indicates that the given quantity is stipulated as increasing; = indicates that the quantity is stipulated as constant; and $\nearrow$ and $\searrow$ indicate the direction of change (increase or decrease, respectively) of the third quantity.

lowing material, I will focus on changes in evidence as data accumulate. It might appear that this is a separate topic from measurement of evidence in a single set of data. But in general, to define the meaning of a unit of some quantity is the same thing as defining the amount of change inherent in adding that unit to another. E.g., $1 \mathrm{~cm}$ always means the same thing with respect to length precisely because adding $1 \mathrm{~cm}$ to any length always increases the length by the same amount. Thus by focusing on changes in evidence with changing data, I am also talking about 'degrees' of evidence measured in a single data set. 


\section{The 1st Law of Thermodynamics and the Likelihood Principle}

The 1st Law of thermodynamics says that $\Delta \mathrm{U}=\mathrm{Q}-\mathrm{W}$, where $\mathrm{U}$ is internal energy, $\mathrm{Q}$ is heat and $\mathrm{W}$ is mechanical work. In this section, I will show how to write the familiar likelihood principle in exactly this same form, substituting in different interpretations for the constituent terms.

The likelihood principle states that all of the information conveyed by a set of data regarding a parameter (vector) is contained in the likelihood. Because below I will need to distinguish this type of information from another, I refer to the information conveyed by the likelihood as 'evidential information'. The other type of information, which I shall call 'combinatoric information', is introduced in the next section.

To be concrete, again consider an experiment comprising $n$ independent coin tosses with $x=$ the number of $\mathrm{H}$, and $\mathrm{p}(\mathrm{H})=\theta$. The likelihood $(\mathrm{L})$ is defined as

$$
\mathrm{L}(\theta \mid n, x) \propto \mathrm{p}_{n}(x \mid \theta)=k \theta^{x}(1-\theta)^{(n-x)},
$$

where $k$ is an arbitrary scaling constant. We are interested in comparing the hypotheses $\theta<1 / 2$ (coin is biased toward $\overline{\mathrm{H}}$ ) versus $\theta=1 / 2$ (coin is fair). Then the likelihood principle further implies that all of the information distinguishing these hypotheses for given data $(n, x)$ is contained in the likelihood ratio (LR) between these two hypotheses:

$$
\operatorname{LR}(\theta \mid n, x)=\frac{k \theta^{x}(1-\theta)^{(n-x)}}{k\left(\frac{1}{2}\right)^{n}}=2^{n} \theta^{x}(1-\theta)^{(n-x)} .
$$

I will refer to this extrapolation of the likelihood principle from the likelihood itself to the LR as the extended likelihood principle. Note that equation 2 is frequently treated in the statistical literature as expressing the evidence for (or against) 'coin is biased' versus 'coin is fair' (see, e.g., Hacking [15], Edwards [16] and Royall [17]), but the line of reasoning pursued here will lead to a fundamentally different interpretation. (In general, when equation 2 is used as a measure of evidence, it is assumed to be in the form of a simple-versus-simple comparison, e.g., with $\theta$ replaced by its maximum likelihood estimate. But here the LR is treated as a function of $\theta$.)

Now consider two sets of data. Let $\mathrm{D}_{1}$ comprise $n_{1}$ tosses of which $x_{1}$ have landed $H$. Denote the graph of the corresponding $\operatorname{LR}(\theta \mid n, x)$, plotted over $\theta=[0,1 / 2]$ on the $\mathrm{x}$-axis, as $\mathrm{LR}(\mathrm{A})$. We are interested in the effects on $\mathrm{LR}(\mathrm{A})$ of a second data set, $\mathrm{D}_{2}=\left(n_{2}, x_{2}\right)$. Let the corresponding graph, considering both $\mathrm{D}_{1}$ and $\mathrm{D}_{2}$, be $\mathrm{LR}(\mathrm{B})$. The con- sideration of $\mathrm{D}_{2}$ results in a transformation of the graph from its initial state A to a new state B. Even prior to considering the nature or mechanism of that transformation, one requirement is that it must appropriately reflect the effects of the new data and nothing but the effects of the new data. Otherwise, the transformation would lead to a violation of the extended likelihood principle. Thus we will need to characterize transformations of the LR graph in terms of an underlying state variable, which I shall denote by $U$. Requiring $U$ to be a state variable means that, for any given set of data $(n, x), \mathrm{U}$ must depend only on the LR for the given data, and not, for example, on anything related to the history of data collection.

I now seek to formally characterize the change in $\mathrm{U}$ from state $\mathrm{A}$ to state $\mathrm{B}$, or $\Delta \mathrm{U}$, corresponding to the change $\Delta \mathrm{LR}$. The binomial LR graph for a given $(n, x)$ can be uniquely specified in terms of two quantities, but there is leeway regarding which two we choose. For example, we could use $(n, x)$ itself, but this turns out to be not particularly revealing in the current context. Alternatively, we can uniquely specify the LR in terms of properties of the LR graph, where again we have a choice of many possible pairs of quantities. Here I use the area under the LR curve, denoted by $\mathrm{V}$, and a second quantity denoted by $\mathrm{P}$. P is chosen such that the pair $(\mathrm{V}, \mathrm{P})$ uniquely determines a particular LR graph, and such that for given $U, P$ is inversely proportional to $\mathrm{V}$. (The existence of a quantity $\mathrm{P}$ fulfilling these conditions in the binomial case is shown in Vieland et al. [6].) For the moment there is no special significance to the choice of variable names for these quantities. However, as a mnemonic device, the reader may consider them as counterparts of volume (V) and pressure (P), respectively.

To consider a concrete example of the bookkeeping involved in quantifying $\Delta \mathrm{U}$, suppose that $\mathrm{D}_{1}=(n, x)=(4$, $1)$ and $D_{2}=(2,1)$. Thus $\operatorname{LR}(A)$ corresponds to $(4,1)$, while $\mathrm{LR}(\mathrm{B})$ corresponds to $(6,2)$. There are two possibilities regarding how we may have gotten from $L R(A)$ to $L R(B)$ : either the 5th toss landed $\mathrm{H}$ and the 6th toss landed $\overline{\mathrm{H}}$, or the 5th toss landed $\overline{\mathrm{H}}$ and the 6th toss landed $\mathrm{H}$. That is, the transformation from $\mathrm{LR}(\mathrm{A})$ to $\mathrm{LR}(\mathrm{B})$ could go through the intermediate states $(5,1)$ or $(5,2)$. Call these two possibilities path 1 and path 2, respectively. Figure 2 shows the two paths plotted both in terms of the LR and on a classical physics PV diagram. (Details of calculations are shown in Appendix 1.)

Since both paths begin and end with identical LR graphs, our state variable $U$ must change by the same amount in both cases. Thus we would like to find a function of $\mathrm{V}, \mathrm{P}$ such that $\mathrm{dU}(\mathrm{V}, \mathrm{P})$ is a perfect differential. By 

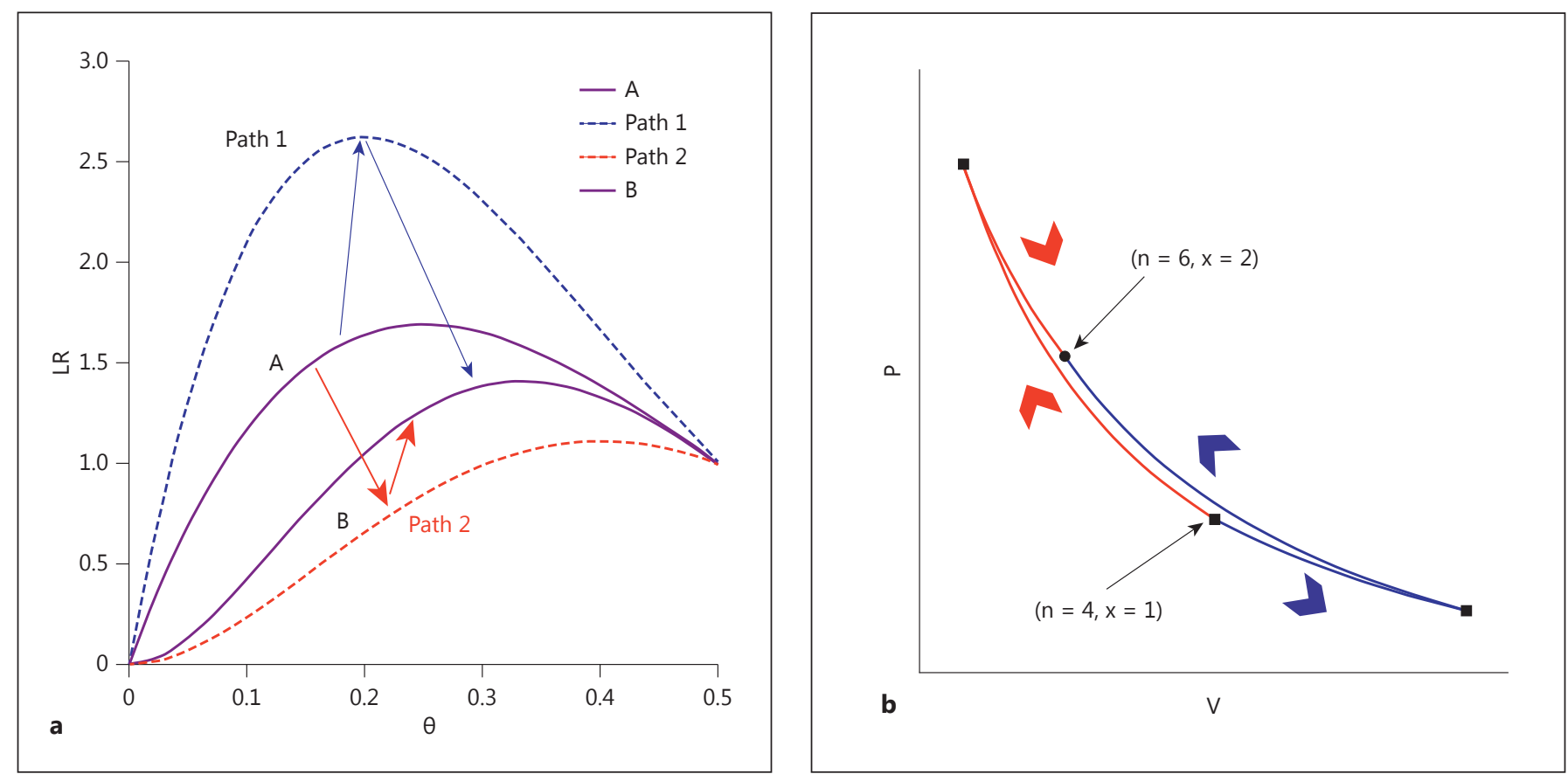

Fig. 2. Path-dependency of transformations of the LR graph shown in terms of the LR itself (a) and projected onto the PV plane by representing each LR by its corresponding (unique) values of $\mathrm{P}$ and V (b). Arrows indicate the direction of 'movement' for the given transformation.

stipulation, $\mathrm{VP} \propto \mathrm{U}$, so that $\mathrm{dU}=\mathrm{c}(\mathrm{VdP}+\mathrm{PdV})$ has the correct form, where $\mathrm{c}$ is the constant of proportionality. Define the quantities $\mathrm{W}=\mathrm{PdV}$ and $\mathrm{Q}=\mathrm{cVdP}+(\mathrm{c}+1)$ $\mathrm{PdV}$. Note that both $\mathrm{W}$ and $\mathrm{Q}$ are readily verified to be path-dependent, i.e., their values will be different for the two transformations. Then we have

$$
\mathrm{dU}=\mathrm{c}(\mathrm{VdP}+\mathrm{PdV})=\mathrm{cVdP}+(\mathrm{c}+1) \mathrm{PdV}-\mathrm{PdV}=\mathrm{Q}-\mathrm{W} .
$$

Beyond the choice of variable names, nothing whatsoever in the derivation makes any reference to physical quantities. Here $\mathrm{W}$ is simply one aspect of the transformation from $\mathrm{LR}(\mathrm{A})$ to $\mathrm{LR}(\mathrm{B})$, and $\mathrm{Q}$ is the compensatory quantity required to express changes in $U$ (a state variable) in terms of W (a path-dependent variable). Then equation 3 tells us that the amount of evidential information 'received' by the $L R$ as a result of new data is $(\mathrm{Q}-\mathrm{W})$.

Armed with this new notation, we now have a concise way to express the principle that all of the evidential information is contained in the LR: $\Delta \mathrm{U}=\mathrm{Q}-\mathrm{W}$, or equivalently, $\mathrm{Q}=\Delta \mathrm{U}+\mathrm{W}$. This is formally identical to the standard representation of the 1st Law of Thermodynamics. We see therefore that the extended likelihood principle can be reformulated to state that the variation in evidential information of a system during any transfor-

Evidence, Temperature, and the Laws of Thermodynamics mation $(\Delta \mathrm{U})$ is equal to the amount of evidential information that the system receives from new data $(\mathrm{Q}-\mathrm{W})$. (This statement is paraphrased from Fermi's general statement of the 1st Law of Thermodynamics [18], p 11.)

In physics, the constituent quantities referred to in the 1st Law of Thermodynamics are inherently interpreted with respect to corresponding properties of the physical world (energy, heat and work). To derive the 1st Law of Thermodynamics through a purely mathematical exercise in elementary calculus might thus appear meaningless. However, what this shows is that the extended likelihood principle, which requires that transformations of the LR graph (up to allowable scale transformations) reflect all and only changes in the data, is in fact a fundamental conservation principle on a par with the 1 st Law of Thermodynamics.

\section{The 2nd Law of Thermodynamics and Information Loss}

The 2nd Law of Thermodynamics has the peculiarity of being notoriously hard to pin down, considering how fundamental it is. It can be expressed in many different ways, and even among physicists its interpretation is sub- 
ject to debate. Here I build up to one form of the 2nd Law of Thermodynamics, which coincides with at least one version of the physical principle, in a way to be made explicit in what follows. I continue with the coin-tossing example. But whereas the previous section dealt with what I called 'evidential information', I now consider another type of information (combinatoric information).

In a sequence of $n$ independent tosses of a single coin, each toss can land one of two ways, either $\mathrm{H}$ or $\overline{\mathrm{H}}$. Hence we have $\log 2$ units of information for each observed toss. Then if we know the actual sequence of tosses, we have

$$
\mathrm{I}_{\mathrm{SEQ}}=\log 2^{n}=n \log 2
$$

units of information for the full set of $n$ tosses. (For present purposes the base of the logarithm, which further defines the units of information, is unimportant. Note that equation 4 is more commonly thought of as the amount of uncertainty or entropy associated with having no knowledge of the sequence; but it will be more convenient here to consider this as the information corresponding to complete knowledge of the sequence.) Obviously $\mathrm{I}_{\mathrm{SEQ}}$ increases linearly with $n$. This makes sense: all other things being equal, the amount of information goes up with the number of tosses.

Now suppose that, rather than knowing the full sequence of $\mathrm{H}$ and $\overline{\mathrm{H}}$, we know only the number of $\mathrm{H}(x$; and hence the number of $\overline{\mathrm{H}}: n-x)$. In this case, we know only that the actual sequence was one of

$$
\left(\begin{array}{l}
n \\
x
\end{array}\right)
$$

possibilities, and the amount of information we have is

$$
\mathrm{I}_{(n, x)}=\log \left(\begin{array}{l}
n \\
x
\end{array}\right)<\mathrm{I}_{\mathrm{SEQ}} .
$$

The change in information in going from $\mathrm{I}_{\mathrm{SEQ}}$ to $\mathrm{I}_{(n, x)}$, which is written with a negative sign to indicate lost information, is

$$
-\Delta \mathrm{I} \triangleq \mathrm{I}_{\mathrm{SEQ}}-\mathrm{I}_{(n, x)} \text {. }
$$

E.g., to continue with the concrete example from the previous section, and using units of natural logarithms, for $\mathrm{D}_{1}=(4,1)$ we have $-\Delta \mathrm{I}=4 \log 2-\log 4=1.39$, for $\mathrm{D}_{2}=$ $(2,1)-\Delta \mathrm{I}=2 \log 2-\log 2=0.69$, and for the combined data set $(6,2)$ we have $-\Delta \mathrm{I}=6 \log 2-\log 15=1.45$.

Clearly the behavior of $-\Delta \mathrm{I}$ is a function of both $x$ and $n$. It is not guaranteed to increase as $n$ increases, because the inherent increase in information as $n$ increases is offset by a corresponding increase in the amount of information lost in going from $\mathrm{I}_{\mathrm{SEQ}}$ to $\mathrm{I}_{(n, x)}$, which is further mediated by the value of $x / n$. Thus the underlying dynamics here requires an accounting system that simultaneously takes changes in $n$ and in $x$ into account.

I would argue that this particular type of combinatoric information loss is a ubiquitous feature of abstract reasoning, or the generalization from 'raw' information to explanatory principles. For instance, suppose that what we are really interested in is information about the probability $\theta$ that the coin lands $H$. In order to extract this information about $\theta$, we need to rewrite the sequence of $\mathrm{H}$ and $\overline{\mathrm{H}}$ as an expression in terms of $\theta$. This entails a compression of the original sequence into the expression $\theta^{x}$ $(1-\theta)^{(n-x)}$, or in logarithmic terms,

$$
\mathrm{g}(\theta)=x \log \theta+(n-x) \log (1-\theta)=\log \mathrm{L}(\theta \mid n, x),
$$

which is simply the ordinary log-likelihood for given data, i.e., the log of equation 1. (Thus information about $\theta$ appears to return us to the evidential information conveyed by the likelihood as discussed in the previous section, while the current section deals with some other type of information; see, e.g., Tishby et al. [19] for a related distinction.) But the combinatoric information associated with equation 7 is $\mathrm{I}_{(n, x)}<\mathrm{I}_{\mathrm{SEQ}}$, because once the sequence of $\mathrm{H}, \overline{\mathrm{H}}$ is reduced to this form, the full sequence can no longer be reconstructed.

This illustrates a very general principle: data reduction for purposes of gleaning information about underlying parameters always comes at a price. Any process that extracts information regarding an underlying parameter (vector) from a set of data entails irrecoverable loss of information, or the permanent erasure of some of the information associated with the full data prior to compression. (Of course, had the full sequence been recorded elsewhere, it would still be recoverable.) This is in essence the form of the 2nd Law of Thermodynamics proposed by Duncan and Semura [11], to which I have added a specific context in which information is routinely erased, a context in which we can talk about information loss in accordance with the 2nd Law of Thermodynamics without having to postulate physical existence for the information or for the information lost through data reduction.

Of course the notion of combinatoric information as deployed here probably needs to be generalized, since combinatorics per se does not play the same role in continuous probability distributions or some other discrete distributions. Nevertheless, as soon as we compress a full set of observations into, say, the sample average of a continuously distributed random variable, we have irrevocably lost some of the information associated with the orig- 
inal data, and the exact set of original values cannot be recovered from knowledge of the average alone. Thus the process considered here appears quite general, although some of this discussion has been specific to the multinomial class of likelihoods.

\section{The Missing Link}

Finally, I consider the connection between transformations of the LR graph characterized in terms of changes in evidential information ( $\Delta U$ from The 1st Law of Thermodynamics and the Likelihood Principle) and changes in combinatoric information $(\Delta \mathrm{I}$ from The 2 nd Law of Thermodynamics and Information Loss). A picture emerges of a complex information-dynamic system. All other things being equal, the more data we have, the more evidential information we have. But this information gain is mediated by a corresponding increase in the amount of combinatorial information erased in the process, which is a function (in the binomial case) of both $n$ and $x$. At the same time, while the amount of evidential information is increasing, $e$ itself is not necessarily increasing, because it again is a function of both $n$ and $x$. E.g., evidence in favor of the hypothesis that the coin is biased could decrease going, say, from $(n=4, x=0)$ to $(n=8, x=4)$, despite the doubling of the sample size. Both evidential information and combinatoric information are in play, and this suggests that we need a way to link the bookkeeping expressed in terms of combinatoric information loss with the bookkeeping expressed in terms of evidential information. The only remaining step, then, is to articulate this link.

Following Duncan and Semura [11], I do this by introducing a quantity $\mathrm{E}$, the analogue of thermodynamic temperature $\mathrm{T}$, as the proportionality factor linking the two sets of books. I.e., I postulate that $\mathrm{E}$ relates $\mathrm{U}, \mathrm{Q}$ and $\Delta \mathrm{I}$, as these quantities are defined above in terms of information, through the same equation used to relate $\mathrm{T}$ to the corresponding quantities in thermodynamics. Thus following Duncan and Semura [11], but writing E instead of $\mathrm{T}$, we have

$$
\Delta \mathrm{Q}=-k \mathrm{E} \Delta \mathrm{I},
$$

where $k$ is a constant (not necessarily equal to Boltzmann's constant). Equation 8 relates the incoming evidential information transferred in the form of $\mathrm{Q}$ to the net loss of combinatoric information.

But what reason do we have for thinking that the relationship expressed between the two sides of equation

Evidence, Temperature, and the Laws of

Thermodynamics
8 has any useful meaning, given that we are not interpreting Q as physical heat? In the current context, E plays a purely abstract role, as the link relating evidential and combinatoric information. Of course, Kelvin's derivation of T was also quite abstract, arguably a matter more of calculus than physics, and historically predating our understanding of thermal energy in the terms of statistical mechanics. (For a fascinating account of exactly how difficult it was, and remains to this day, to directly relate $\mathrm{T}$ to actual physical phenomena, see Chang [20], especially chapter 4.) Notwithstanding, T itself has a critically important physical interpretation in the theory of thermodynamics. It behooves us, therefore, to seek a corresponding information-based interpretation of E.

Figure 3 illustrates the behavior of $\mathrm{E}$, as defined by equation 8 , as a function of $(n, x)$. (See Appendix 1 for calculation details.) It is readily confirmed that in its behavior, E recapitulates the principal properties ascribed to evidence in the section Evidence (per table 1). Thus E can be understood to be the evidence measured on an absolute scale. (I.e., $\mathrm{E}$ is the analogue of $\mathrm{T}$, while the quantity $e$ in the section Evidence is the analogue of thermodynamic $t$, or temperature measured on an arbitrary scale. See also Vieland et al. [6] for additional detail on E as a formal measure of evidence.) Thus it is possible to align thermodynamics with this purely informationbased framework, through both the new interpretations given above for the 1st and 2nd Law of Thermodynamics as well as through the inclusion of this new quantity E, which appears in the end as the thing the new theory is actually about: the evidence.

\section{Conclusions}

Here I have derived a conceptual framework in which the 1st Law of Thermodynamics, the 2nd Law of Thermodynamics and even $\mathrm{T}$ itself - here renamed $\mathrm{E}$ - play their usual thermodynamic roles without the need to posit physical existence for any of the underlying quantities. Of course, I am not proposing to replace the original physical understanding of the laws. But the particular interpretations assigned by thermodynamics may be just one among multiple coherent and potentially fruitful ways to view systems that operate under governance of equations in the form of thermodynamic laws. Under the entirely information-based interpretation proposed here, these laws set the stage for a new look at statistical inference and, more generally, any type of mathematical modeling, the 

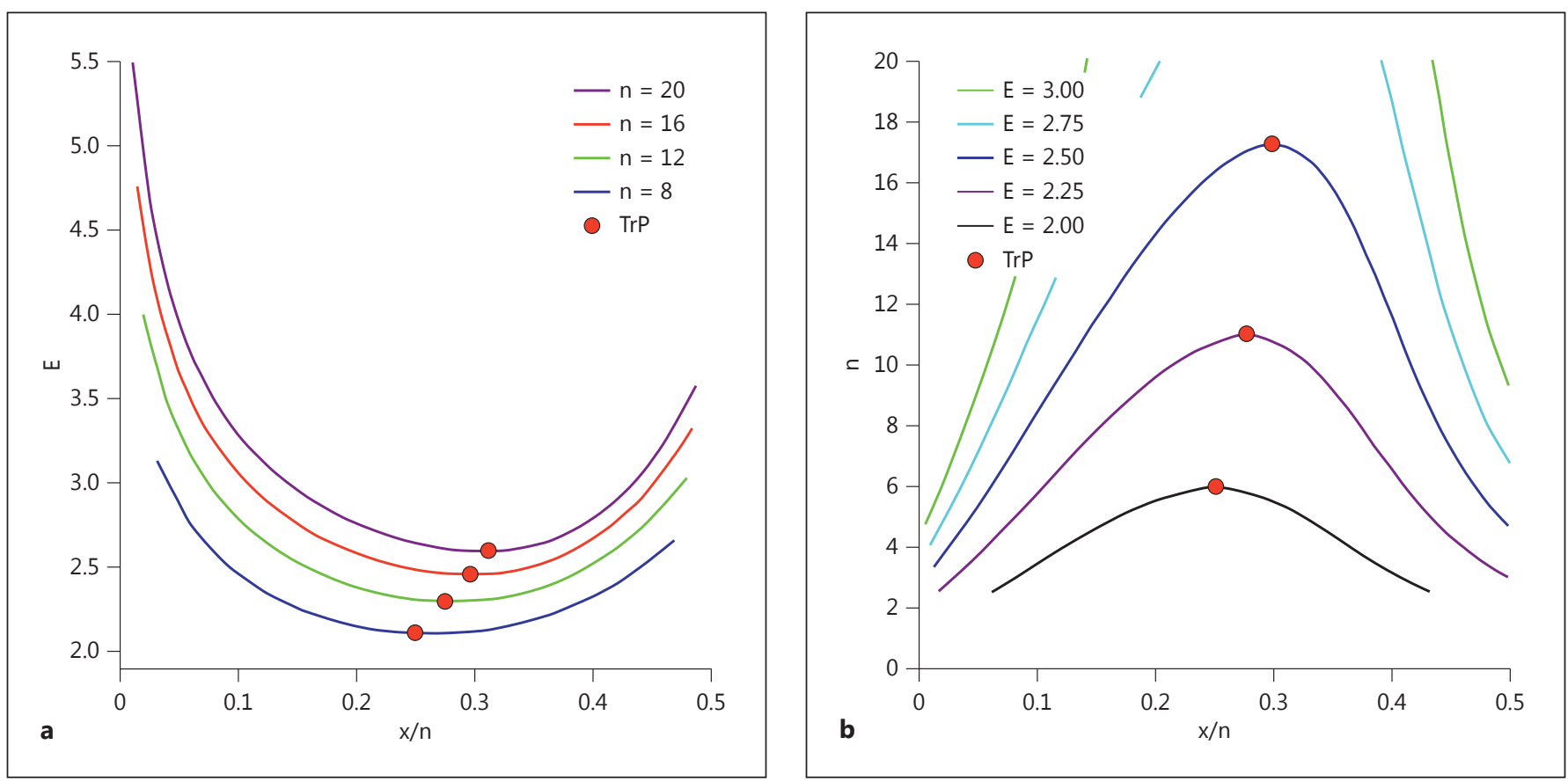

Fig. 3. Correspondence between the behavior of $\mathrm{E}$ and the properties P1-P4 of evidence $e$ as shown in table 1: a the behavior of $\mathrm{E}$ as a function of $x / n$ for different values of $n$, displaying properties P1-P2; b 'isotherms' of the evidential system (holding E constant), displaying property $\mathrm{P} 3$; $\mathbf{c}$ changes in $\mathrm{E}$ as a function of increasing $n$, starting with the $(n, x)$ pair $(1,0)$ and increasing by one tail at a time [i.e., going from $(1,0)$ to $(2,0)$ to $(3,0) \ldots$ ], illustrating property $\mathrm{P} 4$.

aim of which is to assess evidence for or against various models or hypotheses.

I want to stress that in terms of the theory as it has been derived here, the relationship between $\mathrm{E}$ and evidence as shown in The Missing Link is an empirical discovery. E was introduced as the link connecting two types of information bookkeeping, assuming a system governed by

purely information-based versions of the 1st and 2nd Law of Thermodynamics, and under the postulate that $\mathrm{E}$ would have the same mathematical form as its analog $\mathrm{T}$ in thermodynamics. E need not have turned out to have any recognizable behavior or meaningful interpretation. The fact that it turns out to have an interpretation as evidence, a concept so seemingly fundamental to inference, 
strongly supports the proposition that thermodynamics is serving here as much more than mere analogy. The mathematical underpinnings of thermodynamics appear to relate to the 'dynamics' of information flow just as directly as they relate to heat flow. Indeed, from this new perspective it might appear to be $\mathrm{T}$ - rather than $\mathrm{E}$ - that is the more mysterious quantity. Had 'evidentiodynamics' preceded thermodynamics historically, we would undoubtedly be resistant to the notion that the proportionality factor connecting two forms of information bookkeeping could have purely physical existence, let alone existence as the basis for what we feel when we experience changes in temperature.

One immediate implication of this perspective for statistical geneticists relates to the LR, which is usually interpreted as representing the statistical evidence. In many contexts, this interpretation is assigned to the max $L R=$ $\operatorname{LR}(\hat{\theta})$ (to continue for notational simplicity with our binomial example having a single free parameter in the numerator of the LR and none in the denominator); in others an interpretation in terms of evidence is assigned to the Bayesian quantity $\int \operatorname{LR}(\theta) \pi(\theta) d \theta$, where $\pi(\theta)$ is the prior distribution. In the current framework, however, $\operatorname{LR}(\hat{\theta})$ would appear to be better understood as a measure of information loss or relative entropy (see Appendix 2), while $\int \operatorname{LR}(\theta) d \theta$ plays the role of $V$, not evidence. (See also, e.g., Kullback [21], Zellner [22] and Soofi [23] for other accounts in which the LR is considered in information-theoretic terms.) Furthermore, the simple-versuscomposite form of hypothesis contrast considered here may prove to be fundamental, insofar as this particular form of LR can also be described as comparing the unconstrained maximum entropy 'state' of the system (for the binomial model, the entropy is maximized at $\theta=1 / 2$ ) with the entropy constrained by the observed data (see Appendix 2). Thus in several ways the new framework is difficult to reconcile directly with much of the familiar literature on the foundations of statistical inference. This is perhaps not so surprising, since it also differs from most of that literature in its foundational emphasis on evidence and measurement, rather than the more familiar statistical objectives of parameter estimation, hypothesis testing, quantification of statistical error rates, and/or the rational rank ordering of beliefs. It is also interesting to note that here I have considered entropy as a function of a particular, observed set of data (see also Vieland et al. [6]). This is different from Shannon entropy (as deployed by Jaynes [8] and Kullback [21]), which is by definition averaged across all possible data sets. Interestingly, Duncan and Semura [12] also stressed the importance of 'observed' rather than average entropy in their reinterpretation of the 2nd Law of Thermodynamics.

So much of what we do in genetics depends upon reliable measurement of the strength of evidence based on available data. The importance of ensuring a consistent measurement scale only increases as we move into the era of 'big data' and systems-level analyses, where the rigorous accumulation of evidence requires measurement in constant units across distinct data sets and different types of data. Thus despite the abstract, philosophical nature of the topic, it behooves us to get very serious about how we measure evidence. It is a tough problem, but it looks like physics is prepared to lend us a hand in working out a solution. That seems like an offer we cannot afford to refuse.

\section{Appendix 1}

\section{Methods Used in the Calculations}

Numerical calculations used in generating the figures were done as described in detail in Vieland et al. [6]. Briefly, let us begin with the LR (equation 2), and two key features of the LR graph. First, let us define the area under the LR as

$$
\mathrm{V}=\int \operatorname{LR}(\theta) \mathrm{d} \theta=\int 2^{n} \theta^{x}(1-\theta)^{(n-x)} \mathrm{d} \theta .
$$

Here the integral is taken over the interval $\theta=[0, \ldots, 1 / 2]$, reflecting the one-sided hypothesis contrast considered above. Second, let us treat the maximum $\log$ LR as a form of entropy, or evidential entropy, denoted $\mathrm{S}_{\mathrm{E}}$. From there we simply follow the mathematics of thermodynamics for ideal gases, setting $\mathrm{U}=\mathrm{C}_{\mathrm{V}} \mathrm{E}$, where $\mathrm{C}_{\mathrm{V}}$ is a constant. This entails defining a quantity $\mathrm{P}$ through the relationship PV = RE, where R is another constant, and assuming that the system follows the 1st and 2nd Law of Thermodynamics. Following Fermi [18] (p. 147), this gives us

$$
\mathrm{S}_{\mathrm{E}}=\mathrm{C}_{\mathrm{V}} \log \mathrm{E}+\mathrm{R} \log \mathrm{V} \text {. }
$$

For purposes of these calculations, we set $\mathrm{C}_{\mathrm{V}}$ and $\mathrm{R}$ somewhat arbitrarily to 1.5 and 1, respectively. (Note that changing $C_{V}$ affects the size of the units of $\mathrm{E}$. Note also that this sets the constant in equation 3 to $c=C_{V} / R$. See Vieland et al. [6] for an additional discussion regarding the constants.) This gives us

$$
\mathrm{E}=\frac{\exp \left\{\mathrm{S}_{\mathrm{E}} / \mathrm{C}_{\mathrm{V}}\right\}}{\mathrm{V}^{\mathrm{R} / \mathrm{C}_{\mathrm{V}}}} .
$$

Note that $\mathrm{P}$ can then be calculated from $\mathrm{E}$ and $\mathrm{V}$ as

$$
\mathrm{P}=\frac{\mathrm{RE}}{\mathrm{V}} .
$$

All calculations were done in MATLAB; numerical integrals were computed assuming continuous linear changes in $n$ and $x$.

While the results in the main text do not depend upon particular choices for $\mathrm{V}, \mathrm{P}$ or $\mathrm{S}_{\mathrm{E}}$, of course the specific numerical results 
shown in figures 2 and 3 do depend upon these choices. As noted above, multiple equivalent parameterizations of the binomial evidential model are possible. However, it remains an open question how much latitude exists in choosing specific forms for $\mathrm{V}, \mathrm{P}$ and $\mathrm{S}_{\mathrm{E}}$, while maintaining the overall patterns in the behavior of E exhibited by the current system.

\section{Appendix 2}

\section{LRs, Entropy and Information Loss}

The general form of Shannon entropy for a discrete probability distribution is

$$
\mathrm{S}=-\sum_{x=0}^{n} f(x) \log f(x),
$$

where $f(x)$ is the probability of $x$. To see the connection to the loglikelihood (equation 7), rather than starting with the usual form of the binomial probability distribution, consider the complete set of ordered sequences of $\mathrm{H}$ and $\overline{\mathrm{H}}$. E.g., with $\mathrm{n}=3$, there are $i=$ $2^{3}=8$ such sequences: $\overline{\mathrm{H}} \overline{\mathrm{H}} \overline{\mathrm{H}}, \overline{\mathrm{H}} \overline{\mathrm{H}} \mathrm{H}, \overline{\mathrm{H}} \overline{\mathrm{H}}, \mathrm{H} \overline{\mathrm{H}} \overline{\mathrm{H}}, \overline{\mathrm{H}} \mathrm{H}, \mathrm{H} \overline{\mathrm{H}}$, $\mathrm{HH} \overline{\mathrm{H}}, \mathrm{HHH}$. For each sequence $i$, the probability $f(i)$ equals $\theta^{x}$ $(1-\theta)^{(n-x)}$, where $x$ is the number of $H$ in the sequence as above. Rewriting the summation over $x$ in equation 13 as a summation over $i$ and then collecting like terms, we have

$$
\begin{aligned}
-\mathrm{S} & =\sum_{i=1}^{2^{n}}[f(i) \log f(i)] \\
& =\sum_{i=1}^{2^{n}} \theta^{x}(1-\theta)^{n-x} \times \log \left[\theta^{x}(1-\theta)^{n-x}\right] \\
& =\sum_{x=0}^{n}\left(\begin{array}{l}
n \\
x
\end{array}\right) \theta^{x}(1-\theta)^{n-x} \times \log \left[\theta^{x}(1-\theta)^{n-x}\right] \\
& =\mathrm{E}_{x}[x \log \theta+(n-x) \log (1-\theta)]=\mathrm{E}_{x}[\log \mathrm{L}(\theta \mid n, x)],
\end{aligned}
$$

i.e., the expected value of equation 7 . In other words, if we begin with the log-probabilities of the individual sequences as our fundamental units of information, then the Shannon entropy - generally written as the expected value of a log-probability - becomes the expected value of a log-likelihood.

Maximizing equation 7 yields the familiar maximum likelihood estimate (MLE) $\hat{\theta}=x / n$, and evaluating equation 7 at the MLE reveals an (approximate) equivalence with the combinatoric information expressed in equation 5 (using Stirling's approximation):

$$
\log \mathrm{L}(\hat{\theta} \mid n, x)=x \log x+(n-x) \log (n-x)-n \log n \sim \log \left(\begin{array}{l}
n \\
x
\end{array}\right) .
$$

Evaluating equation 2 at the MLE of $\theta$ in the numerator and taking the $\log$ yields

$$
\begin{aligned}
\log \operatorname{LR}(\hat{\theta}) & =\log \frac{\hat{\theta}^{x}(1-\hat{\theta})^{n-x}}{1 / 2}=\log \frac{\left(\frac{x}{n}\right)^{x}\left(\frac{n-x}{n}\right)^{n-x}}{1 / 2} \\
& =x \log x+(n-x) \log (n-x)-n \log n+n \log 2 .
\end{aligned}
$$

Now if we also evaluate equation 13 at the MLE, which is also the value of $\theta$ that maximizes the Shannon entropy, and assume 'perfect' data or data occurring in their exact expected proportions (as it would occur for very large values of $n$ ), then we have

$$
-\mathrm{S}(\hat{\theta})=x \log x+(n-x) \log (n-x)-n \log n,
$$

so that

$$
\log \operatorname{LR}(\hat{\theta})=-\mathrm{S}(\hat{\theta})+n \log 2=-\Delta \mathrm{I} .
$$

This has implications for the way we interpret the max log $\operatorname{LR}(\theta)$, which is frequently taken to be a measure of statistical evidence. Equation 18 suggests instead that it might be more appropriately understood as a measure of relative information.

\section{Acknowledgements}

This paper was written in the context of an ongoing collaboration with J. Das, S.E. Hodge and S.-C. Seok, all of whom have influenced the development of the theory and provided valuable commentaries on drafts of this paper. S.-C. Seok performed the calculations. An earlier version containing additional technical information and discussion was posted to arXiv:1301.2150. I am also indebted to Hasok Chang and Gunter Wagner for their willingness to invest effort in following this work and for their many helpful critical suggestions, which have propelled the project forward.

\section{References}

1 Vieland VJ: Thermometers: something for statistical geneticists to think about. Hum Hered 2006;61:144-156.

12 Vieland VJ: Where's the evidence? Hum Hered 2011;71:59-66.

- 3 Hodge SE, Vieland VJ: Expected monotonicity - a desirable property for evidence measures? Hum Hered 2010;70:151-166.

4 Vieland VJ, Huang Y, Seok SC, Burian J, Catalyurek U, O'Connell J, Segre A, Valentine-Cooper W: KELVIN: a software package for rigorous measurement of statistical evidence in human genetics. Hum Hered 2011; 72:276-288.
5 Vieland VJ, Hodge SE: Measurement of evidence and evidence of measurement. Stat App Genet Mol Biol 2011;10:Article 35.

6 Vieland VJ, Das J, Hodge SE, Seok SC: Measurement of statistical evidence on an absolute scale following thermodynamic principles. Theory Biosci 2013;132:181-194.

$\checkmark 7$ Shannon CE: A mathematical theory of communication. Bell Syst Tech J 1948;27:379423, 623-656.

8 Jaynes ET: The Gibbs paradox; in Smith CR, Erickson G, Neudorfer PO (eds): Maximum Entropy and Bayesian Methods. Dordrecht, Kluwer Academic Publishers, 1992. 
9 Szilard L: On the decrease of entropy in a thermodynamic system by the intervention of intelligent beings. Z Phys 1929;53:840-856.

10 Landauer R: Irreversibility and heat generation in the computing process. IBM J Res Dev 1961;5:183-191.

11 Duncan TL, Semura JS: The deep physics behind the second law: information and energy as independent forms of bookkeeping. Entropy $2004 ; 6: 21-29$.

12 Duncan TL, Semura JS: Information loss as a foundational principle for the second law of thermodynamics. Found Phys 2007;37:17671773.
13 Caticha A: Relative Entropy and Inductive Inference. http://arXiv/0311093v1, 2003.

14 Caticha A: From Inference to Physics. http:// arxiv.org/pdf/0808.1260v1, 2008.

15 Hacking I: Logic of Statistical Inference. London, Cambridge University Press, 1965.

16 Edwards A: Likelihood. Baltimore, Johns Hopkins University Press, 1992.

17 Royall R: Statistical Evidence: A Likelihood Paradigm. London, Chapman \& Hall, 1997.

18 Fermi E: Thermodynamics. New York, Dover, 1956 (orig. 1936).
19 Tishby N, Pereira FC, Bialek W: The Information Bottleneck Method. http://arXiv: 0004057, 2000.

20 Chang H: Inventing Temperature: Measurement and Scientific Progress. New York, Oxford University Press, 2004

21 Kullback S: Information Theory and Statistics. Mineola, Dover, 1968 (orig. John Wiley \& Sons, 1959).

22 Zellner A: Optimal information processing and Bayes's theorem. Am Statistician 1988;42: 278-280.

23 Soofi ES: Principal information theoretic approaches. J Am Statist Assoc 2000;95:13491353. 